\title{
The Risk of Upper Urinary Tract Involvement in Patients With Ketamine-Associated Uropathy
}

\author{
Chi-Hang Yee ${ }^{1}$, Jeremy Yuen-Chun Teoh ${ }^{1}$, Pui-Tak Lai ${ }^{1}$, Vivian Yee-Fong Leung ${ }^{2}$, Winnie Chiu-Wing Chu ${ }^{3}$, Wai-man Lee ${ }^{1}$, \\ Yuk-Him Tam ${ }^{4}$, Chi-Fai $\mathrm{Ng}^{1}$ \\ ${ }^{1}$ Prince of Wales Hospital, SH Ho Urology Centre, Department of Surgery, The Chinese University of Hong Kong, Hong Kong \\ ${ }^{2}$ Department of Imaging and Interventional Radiology, Prince of Wales Hospital. Hong Kong \\ ${ }^{3}$ Department of Imaging and Interventional Radiology, Prince of Wales Hospital, The Chinese University of Hong Kong. Hong Kong \\ ${ }^{4}$ Prince of Wales Hospital, Department of Surgery, The Chinese University of Hong Kong. Hong Kong
}

Purpose: The aims of this study were to investigate the prevalence of upper tract involvement in ketamine-associated uropathy, and to determine the predictors of hydronephrosis in patients with a history of ketamine abuse.

Methods: This was a cross-sectional study of a prospective cohort of patients with ketamine-associated uropathy. Data including demographics, pattern of ketamine abuse, pelvic pain and urgency or frequency (PUF) symptom score, uroflowmetry (UFM) parameters, serum renal function, and liver function tests were collected. Upon consultation, ultrasonography was performed to assess the function of the urinary system.

Results: From December 2011 to October 2015, we treated 572 patients with ketamine-associated uropathy. Of these patients, $207(36.2 \%)$ had managed to achieve abstinence at the time of their first consultation. Ninety-six patients (16.8\%) in the cohort were found to have hydronephrosis on ultrasonography. Univariate analysis identified age, duration of ketamine abuse, PUF symptom score, voided volume on UFM, serum creatinine levels $>100 \mu \mathrm{mol} / \mathrm{L}$, and an abnormal serum liver enzyme profile as factors associated with hydronephrosis. Logistic regression revealed the following parameters to be statistically related to hydronephrosis: age (adjusted odds ratio [OR], 1.090; 95\% confidence interval [CI], 1.020-1.166; P=0.012), functional bladder capacity (adjusted OR, 0.997; 95\% CI, 0.995-0.999; P=0.029), serum creatinine > $100 \mu \mathrm{mol} / \mathrm{L}$ (adjusted OR, 3.107; 95\% CI, 1.238-7.794; $\mathrm{P}=0.016$, and an abnormal serum liver enzyme profile (adjusted OR, 1.967; 95\% CI, 1.213-3.187; $\mathrm{P}=0.006)$.

Conclusions: Ketamine-associated uropathy can involve the upper urinary tract. Patient demographics as well as investigations of UFM, renal function tests, and liver function tests may allow us to identify at-risk patients.

Keywords: Cystitis; Hydronephrosis; Ketamine; Lower Urinary Tract Symptoms; Urination Disorders

- Fund/Grant Support: The research project was funded by the Beat Drugs Fund, The Government of the Hong Kong Special Administrative Region.

- Research Ethics: This study was approved by the Joint Chinese University of Hong Kong - New Territories East Cluster Clinical Research Ethics Committee (CREC No. CRE-2011.454).

- Conflict of Interest: No potential conflict of interest relevant to this article was reported.

Corresponding author: Chi-Hang Yee (iD https://orcid.org/0000-0001-6557-3107 Prince of Wales Hospital, SH Ho Urology Centre, Department of Surgery, The Chinese University of Hong Kong, 30-32 Ngan Shing Street, Shatin, New Territories, Hong Kong

E-mail: yeechihang@surgery.cuhk.edu.hk / Tel: +852-26322625 / Fax: +852-26377974 Submitted: August 17, 2016 / Accepted after revision: September 29, 2016
This is an Open Access article distributed under the terms of the Creative Commons Attribution Non-Commercial License (http://creativeicenses/by-nc/4.0/) which permits unrestricted non-commercial use, distribution, and reproduction in any medium, provided the original work is properly cited. 


\section{INTRODUCTION}

Ketamine has been used for anaesthetic and analgesic purposes since the 1960s [1]. It is an N-methyl-D-aspartate antagonist and is metabolized in the liver to norketamine. This active metabolite of ketamine is then excreted in the urinary system. In addition to its role in pain relief, ketamine has been adopted as a treatment option for major depression [2], treatment-resistant depression [3], and bipolar affective disorder [4]. However, since it has the properties of a strong psychostimulant, ketamine can also be a drug of abuse. The recreational use of ketamine was first reported in the 1970s, and it has become increasingly common in the past 20 years [5]. An increase in ketamine use from $0.8 \%$ in $2007-2008$ to $2.1 \%$ in $2010-2011$ was noted among young people aged 16-24 years in the United Kingdom [6], and it has been the substance most commonly abused by teenagers since 2005 in Asian cities such as Hong Kong [7].

Ketamine is known to be toxic to the urinary tract. Since the initial case series of ketamine cystitis reported by Shahani et al. [8] and Chu et al. [9] in 2007, this unique disease entity has gained increasing recognition. The original report by $\mathrm{Chu}$ et al. [9] described 59 patients with a history of ketamine abuse who presented with lower urinary tract symptoms. Their report demonstrated that ketamine-associated uropathy involves both the lower urinary tract and the upper urinary tract. Thirty patients (51\%) in their series had unilateral or bilateral hydronephrosis on renal ultrasonography. In another cohort of $160 \mathrm{pa}-$ tients with ketamine-associated uropathy presented by Tam et al. [10], 8.1\% of the patients had hydronephrosis. We investigated the prevalence of hydronephrosis in an updated cohort of patients with ketamine-associated uropathy, and determined the predictive factors of hydronephrosis.

\section{MATERIALS AND METHODS}

Since December 2011, all consecutive patients who attended our centre for ketamine-related urological problems were seen in a dedicated clinic, and were recruited into a prospective cohort with a standardized treatment regimen after we obtained approval from the Joint Chinese University of Hong KongNew Territories East Cluster Clinical Research Ethics Committee (CREC No: CRE-2011.454). Written informed consent was provided by all participants prior to entering the study.

Basic demographic data were recorded before clinic attendance. Upon the first consultation, serum creatinine levels were determined, and urine microscopy and culture and uroflowmetry (UFM), were performed. Functional bladder capacity (FBC) was calculated by adding the voided volume to the postvoid residual urine the UFM, assessment. Symptom assessment was done using the pelvic pain and urgency/frequency symptom scale (PUF symptom score) and the EuroQoL visual analogue scale (EQ VAS). The Chinese version of the PUF symptom score is a validated assessment tool for cystitis [11], and the EQ VAS is a $0-100$ visual scale for the patient's subjective assessment of his or her own health state. The higher the score, the better the patients perceive their health state. Ultrasound of the urinary system was performed to identify signs of obstructive uropathy.

Descriptive statistics were used to characterize the clinical characteristics of the study cohort. Abstinence was defined as abstaining from ketamine use for at least 4 weeks. The t-test was used for continuous data, and the Mann-Whitney U-test was used for skewed data. The chi-square test was applied for categorical data. Univariate and multivariate logistic regression analyses were performed to identify clinical covariates that were significantly associated with a hydronephrotic status. P-values $<0.05$ were considered to indicate statistical significance. IBM SPSS Statistics ver. 21.0 (IBM Co., Armonk, NY, USA) was used for all calculations.

\section{RESULTS}

From December 2011 to October 2015, 572 patients presented to our clinic with ketamine-associated uropathy (Table 1). Female patients comprised a slight majority of our cohort (323 patients; $56.5 \%$ ) and the mean age was $25.3 \pm 3.8$ years. Of the patients, 207 (36.2\%) had already managed to achieve abstinence upon their first consultation, which was defined as abstaining from ketamine use for 4 weeks. Ninety-six patients $(16.8 \%)$ in the cohort were found to have either unilateral or bilateral hydronephrosis on ultrasonography. Among the patients with hydronephrosis, 9 patients required urinary diversion with a percutaneous nephrostomy drain, and 4 patients required double-J stent insertion. Some selected patients were investigated further with an antegrade pyelogram, a retrograde pyelogram, or a technetium-99m diethylenetriamine-pentaacetic acid scan to delineate the cause of hydronephrosis. Among the 19 patients who underwent these investigations, 8 were found to have an obstruction at the ureter, pelvic-ureteric junction, or vesico-ureteric junction. For patients without any demonstrable ureteric obstruction, no video urodynamic study or micturat- 
Table 1. Patient demographics and characteristics with univariate analysis

\begin{tabular}{|c|c|c|c|c|}
\hline Variable & Entire Cohort $(\mathrm{n}=572)$ & Without hydronephrosis $(\mathrm{n}=476)$ & With hydronephrosis $(\mathrm{n}=96)$ & P-value \\
\hline Age (yr) & $25.3 \pm 3.8$ & $25.0 \pm 3.8$ & $26.3 \pm 3.7$ & 0.002 \\
\hline $\begin{array}{l}\text { Sex } \\
\text { Female } \\
\text { Male }\end{array}$ & $\begin{array}{l}323(56.5) \\
249(43.5)\end{array}$ & $\begin{array}{l}264(55.5) \\
212(44.5)\end{array}$ & $\begin{array}{l}59(61.5) \\
37(38.5)\end{array}$ & 0.280 \\
\hline Achieved abstinence & $207(36.2)$ & $175(36.8)$ & $32(33.3)$ & 0.523 \\
\hline Duration of abuse (mo) & $86.8 \pm 37.7$ & $84.6 \pm 37.7$ & $97.6 \pm 35.7$ & 0.002 \\
\hline Amount consumed per week (g) & $20.4 \pm 18.1$ & $20.7 \pm 18.1$ & $18.6 \pm 18.1$ & 0.306 \\
\hline PUF total score & $21.0 \pm 7.6$ & $20.6 \pm 7.7$ & $22.9 \pm 6.8$ & 0.006 \\
\hline Functional bladder capacity (mL) & $139.9 \pm 125.5$ & $147.3 \pm 130.8$ & $103.1 \pm 86.5$ & 0.002 \\
\hline Serum creatinine $>100 \mu \mathrm{mol} / \mathrm{L}$ & $25(4.4)$ & $15(3.2)$ & $10(10.4)$ & 0.001 \\
\hline Deranged serum liver enzymes & $258(45.1)$ & $200(42.0)$ & $58(60.4)$ & $<0.001$ \\
\hline
\end{tabular}

Values are presented as mean \pm standard deviation or number (\%).

PUF, pelvic pain and urgency or frequency.

P-value signifies the difference between patients with and without hydronephrosis.

Table 2. Multivariate forward logistic regression analyses of parameters for hydronephrosis

\begin{tabular}{lcc}
\hline Parameter & Adjusted OR (95\% CI) & P-value \\
\hline Age & $1.090(1.020-1.166)$ & 0.012 \\
Functional bladder capacity & $0.997(0.995-0.999)$ & 0.029 \\
Serum creatinine $>100 \mu \mathrm{mol} / \mathrm{L}$ & $3.107(1.238-7.794)$ & 0.016 \\
Deranged serum liver enzymes & $1.967(1.213-3.187)$ & 0.006 \\
\hline
\end{tabular}

OR, odds ratio; $\mathrm{CI}$, confidence interval.

ing cystograms were performed to confirm the presence of vesico-ureteric reflux. In the whole cohort, $19.8 \%$ of the patients were seen for follow-up investigations. Spontaneous resolution of hydronephrosis was observed in 3 patients after they achieved abstinence for 4,8 , and 12 months respectively.

Abstinence status was not found to be significantly different between patients with or without hydronephrosis (32 [33.3\%] vs. 175 [36.8\%], $\mathrm{P}=0.523$ ). The mean amount of ketamine consumed per week in the hydronephrosis group was $18.6 \pm 18.1 \mathrm{~g}$, which was not significantly different from the corresponding value observed in the nonhydronephrotic group. Univariate analysis identified age, duration of ketamine abuse, PUF symptom score, voided volume on UFM, serum creatinine $>100$ $\mu \mathrm{mol} / \mathrm{L}$, and an abnormal serum liver enzyme profile as factors associated with hydronephrosis. The serum liver enzyme profile used in the current study encompassed alkaline phosphatase, aspartate aminotransferase, and alanine aminotransferase. The normal range of the tests was provided by the laboratory in our centre, which met international standards with periodic quality assurance reviews. Any deviation from the normal range was marked as an abnormal liver enzyme profile.

Multivariate forward logistic regression was performed to identify factors related to hydronephrosis (Table 2). The following parameters were found to be statistically significant predictors of hydronephrosis in patients with ketamine-associated uropathy: age (adjusted odds ratio [OR], 1.090; 95\% confidence interval [CI], 1.020-1.166; $\mathrm{P}=0.012$ ), FBC (adjusted OR, 0.997; 95\% CI, 0.995-0.999; $\mathrm{P}=0.029)$, serum creatinine $>100 \mu \mathrm{mol} /$ L (adjusted OR, 3.107; 95\% CI, 1.238-7.794; $\mathrm{P}=0.016$ ), and an abnormal serum liver enzyme profile (adjusted OR, 1.967; 95\% CI, 1.213-3.187; $\mathrm{P}=0.006$ ).

\section{DISCUSSION}

While ketamine-induced urinary symptoms and pathology have sometimes been referred to as ketamine cystitis in the literature [12], the urological sequelae of ketamine abuse extend beyond the lower urinary tract. In the 59 patients with ketamine abuse reported by Chu et al. [9], 30 (51\%) were found to have either unilateral or bilateral hydronephrosis in the initial assessment. Most of these patients in their case series were found to have hydronephrosis and hydroureters down to the level of the vesico-ureteric junctions on intravenous urograms, implying that upper tract involvement was likely the result of a long-term decrease in bladder compliance. In our series, genuine ureteric obstruction was found in some patients with hy- 
dronephrosis. The obstructing points varied from the vesicoureteric junction to the pelvic-ureteric junction. This suggests that when encountering patients with a history of ketamine abuse who have hydronephrosis, both a video urodynamic study of the lower urinary tract and a dynamic contrast study or dynamic nuclear scan of the upper urinary tract are important for establishing the underlying cause of hydronephrosis.

The histopathological findings of bladder biopsy samples obtained from patients with ketamine-induced cystitis include features of chronic inflammation similar to those found in the ulcer type of interstitial cystitis [8,9]. The urothelium is substantially denuded. The lamina propria shows granulation tissue and congested vessels. Infiltration of eosinophils, lymphocytes, plasma cells, mast cells, and macrophages have been observed in the mucosal and submucosal layers [13]. Having said that, interstitial cystitis can seldom lead to a generalized thickened bladder wall as severe as that is seen in ketamine-associated uropathy. Shahani et al. [8] proposed that a high concentration of ketamine and its metabolites in the urine might induce significant bladder irritation and cause cystitis due to prolonged contact. Furthermore, they may inflict damage on the microvasculature of the bladder, potentially leading to ischaemia and fibrosis. This fibrotic process may take place in the ureter as well, ultimately leading to ureteric obstruction and hydronephrosis. However, fibrotic processes in the bladder would decrease the FBC throughout the disease process [14]. In our study, FBC was found to be a predictor of hydronephrosis in patients with a history of ketamine abuse. This may be explained through the possibility that a fibrotic bladder may reflect disease severity, and also implies that a poorly compliant bladder is associated with a higher risk of vesico-ureteric reflux.

In addition to luminal obstruction of the ureter as a result of ureteric fibrosis, intra-luminal obstruction of the ureter by debris was reported by Selby et al. [15]. They reported a patient with a history of ketamine abuse with hydronephrosis and renal failure. Upon nephrostomy drainage, gelatinous debris was aspirated and was present throughout both pelvicalyceal systems. Analysis of this material revealed ketamine metabolites, cannabinoids, and lignocaine. As ketamine does not usually precipitate in the pelvicalyceal system, the authors postulated that the presence of cannabinoid metabolites or repeated administration with a higher cumulative dose of ketamine might account for the production of this debris, which eventually gave rise to a mechanical obstruction of the upper urinary tract.

Nephrogenic metaplasia has also been discovered in the ure- ter of patients with a history of ketamine abuse. In a nephroureterectomy specimen of a patient with ketamine-associated uropathy, florid nephrogenic metaplasia was found throughout the ureter and extending to the renal pelvis [16]. In our cohort, we also had a patient with similar pathology. A 33-year-old man underwent robotic-assisted laparoscopic augmentation cystoplasty with the reimplantation of the bilateral ureters for ketamine-induced cystitis. After the procedure, the patient resumed ketamine abuse for a period of time. Two years after augmentation cystoplasty, he was found to have left hydronephrosis. Ureteroscopy confirmed multiple nodules in his left ureter. Biopsy of the masses showed fragments of granulation tissue with denuded and ulcerated urothelium, associated with dense mixed inflammatory cell infiltrate. Immunohistochemistry showed features consistent with nephrogenic metaplasia. While such histological findings are a common response to injurious stimuli in the bladder, their occurrence in the ureter has rarely been described in the literature.

Our study identified an abnormal serum liver enzyme profile as a factor predicting the presence of upper urinary tract involvement in ketamine-associated uropathy. Ketamine is known to have an effect on the hepatobiliary system. Liver function derangement has been reported in the literature among patients with a history of ketamine abuse [17]. Ketamine-related cholangiopathy in the form of fusiform dilatation of the common bile ducts without evidence of intrinsic or extrinsic obstruction was observed by Yu et al. [18]. Wong et al. [19] reported that ketamine abuse appeared to lead to common bile duct dilatation, microscopic bile duct injury, and even significant liver fibrosis. In a study using rat models to investigate the histological and biochemical impact of ketamine on the liver, ultrastructural changes were seen in the mitochondria and in the rough endoplasmic reticulum [20]. Furthermore, prolonged use of ketamine caused hepatocellular toxicity and histological changes in hepatocytes in a dose-dependent manner. This might imply that the detection of an abnormal serum liver enzyme profile is related to overall disease severity in the setting of ketamine abuse. Thus, such a finding in a patient would mean a higher risk of upper urinary tract involvement in ketamine-associated uropathy.

Abstaining from ketamine abuse is regarded as an important milestone in treating ketamine-related uropathy [11]. With abstinence, symptom resolution has been observed [21]. After patients with ketamine-induced cholangiopathy have achieved abstinence, improvements have been observed in the dilatation 
of the common bile ducts [18]. In our cohort, we observed that hydronephrosis can likewise resolve after abstinence from ketamine has been attained. Furthermore, such resolution can occur after 1 year of abstinence. The predictive factors for the resolution of hydronephrosis after abstinence require further study to be confirmed.

A major limitation of our study stemmed from the relatively poor compliance of our patients. Of all the patients with hydronephrosis, only $19.8 \%$ of the patients returned for follow-up. As hydronephrosis can result from both obstructive and reflux causes, a larger cohort of patients with hydronephrosis who have completed a comprehensive assessment would allow us to better understand the aetiology of hydronephrosis in the context of ketamine abuse.

In conclusion, while lower urinary tract symptoms are a hallmark of ketamine-associated uropathy, upper urinary tract involvement is not uncommon in patients with a history of ketamine abuse. Age, FBC, serum creatinine level, and an abnormal serum liver enzyme profile were found to be correlated with the risk of hydronephrosis in this group of patients. With abstinence, resolution of hydronephrosis was observed in some patients. Nevertheless, permanent toxicity to the upper urinary tract is still of concern with long-term ketamine abuse.

\section{REFERENCES}

1. Lankenau SE, Sanders B. Patterns of ketamine use among young injection drug users. J Psychoactive Drugs 2007;39:21-9.

2. Walter M, Li S, Demenescu LR. Multistage drug effects of ketamine in the treatment of major depression. Eur Arch Psychiatry Clin Neurosci 2014;264 Suppl 1:S55-65.

3. Wan LB, Levitch CF, Perez AM, Brallier JW, Iosifescu DV, Chang LC, et al. Ketamine safety and tolerability in clinical trials for treatment-resistant depression. J Clin Psychiatry 2015;76:247-52.

4. Best SR. Combined ketamine/transcranial magnetic stimulation treatment of severe depression in bipolar I disorder. J ECT 2014;30: e50-1.

5. Kalsi SS, Wood DM, Dargan PI. The epidemiology and patterns of acute and chronic toxicity associated with recreational ketamine use. Emerg Health Threats J 2011;4:7107.

6. Forster JA, Harrison SC. Ketamine uropathy: rising to the challenges of a new condition. BJU Int 2012;109:1277-8.

7. Yiu-Cheung C. Acute and chronic toxicity pattern in ketamine abusers in Hong Kong. J Med Toxicol 2012;8:267-70.

8. Shahani R, Streutker C, Dickson B, Stewart RJ. Ketamine-associat- ed ulcerative cystitis: a new clinical entity. Urology 2007;69:810-2.

9. Chu PS, Ma WK, Wong SC, Chu RW, Cheng CH, Wong S, et al. The destruction of the lower urinary tract by ketamine abuse: a new syndrome? BJU Int 2008;102:1616-22.

10. Tam YH, Ng CF, Pang KK, Yee CH, Chu WC, Leung VY, et al. Onestop clinic for ketamine-associated uropathy: report on service delivery model, patients' characteristics and non-invasive investigations at baseline by a cross-sectional study in a prospective cohort of 318 teenagers and young adults. BJU Int 2014;114:754-60.

11. Ng CM, Ma WK, To KC, Yiu MK. The Chinese version of the pelvic pain and urgency/frequency symptom scale: a useful assessment tool for street-ketamine abusers with lower urinary tract symptoms. Hong Kong Med J 2012;18:123-30.

12. Misra S, Chetwood A, Coker C, Thomas P. Ketamine cystitis: practical considerations in management. Scand J Urol 2014;48:482-8.

13. Lee CL, Jiang YH, Kuo HC. Increased apoptosis and suburothelial inflammation in patients with ketamine-related cystitis: a comparison with non-ulcerative interstitial cystitis and controls. BJU Int 2013;112:1156-62.

14. Yee CH, Lai PT, Lee WM, Tam YH, Ng CF. Clinical outcome of a prospective case series of patients with ketamine cystitis who underwent standardized treatment protocol. Urology 2015;86: 23643.

15. Selby NM, Anderson J, Bungay P, Chesterton LJ, Kolhe NV. Obstructive nephropathy and kidney injury associated with ketamine abuse. NDT Plus 2008;1:310-2.

16. Hopcroft SA, Cottrell AM, Mason K, Abrams P, Oxley JD. Ureteric intestinal metaplasia in association with chronic recreational ketamine abuse. J Clin Pathol 2011;64:551-2.

17. Wong SW, Lee KF, Wong J, Ng WW, Cheung YS, Lai PB. Dilated common bile ducts mimicking choledochal cysts in ketamine abusers. Hong Kong Med J 2009;15:53-6.

18. Yu WL, Cho CC, Lung PF, Hung EH, Hui JW, Chau HH, et al. Ketamine-related cholangiopathy: a retrospective study on clinical and imaging findings. Abdom Imaging 2014;39:1241-6.

19. Wong GL, Tam YH, Ng CF, Chan AW, Choi PC, Chu WC, et al. Liver injury is common among chronic abusers of ketamine. Clin Gastroenterol Hepatol 2014;12:1759-62.e1.

20. Kalkan Y, Tomak Y, Altuner D, Tumkaya L, Bostan H, Yilmaz A, et al. Hepatic effects of ketamine administration for 2 weeks in rats. Hum Exp Toxicol 2014;33:32-40.

21. Winstock AR, Mitcheson L, Gillatt DA, Cottrell AM. The prevalence and natural history of urinary symptoms among recreational ketamine users. BJU Int 2012;110:1762-6. 